\title{
Avalanche dynamics measurement by pulsed Doppler radar
}

\author{
Helmut Sahreiber, ${ }^{1}$ Walter L. Randed, ${ }^{1}$ Horst Sahaffhauser, ${ }^{2}$ Lambert Rammmer ${ }^{2}$ \\ ${ }^{1}$ Institute of Communications and Wave Propagation, Technical University Graz, Inffeldgasse 12, A-8010 Graz, Austria \\ ${ }^{2}$ Austrian Institute for Avalanche and Torrent Research (AIATR/FBVA), Rennweg 1, A-6020 Innsbruck, Austria
}

\begin{abstract}
This paper presents the application of a pulsed Doppler radar for the measurement of dynamic properties of snow and ice avalanches. The instrument was developed and built by the Institute of Communications and Wave Propagation, Technical University Graz, and is operated by the Austrian Institute for Avalanche and Torrent Research (AIATR), Innsbruck. The data acquired by this radar during field campaigns are used to verify and optimize avalanche models and simulation tools. As well as describing the radar's technical principle, how the dynamic parameters are measured and presented to the user and how these data can be used to accomplish the determination of avalanche velocity and runout distance, the paper deals with the participation of the radar in a successful full-scale snow-avalanche experiment in Ryggfonn, Norway, and evaluates the collected data. This measurement campaign was a joint experiment by the Norwegian Geotechnical Institute, Oslo, and AIATR.
\end{abstract}

\section{IDEA AND TEGHNIGAL PRINGIPLE}

The development of pulsed Doppler avalanche radars at the Institute of Communications and Wave Propagation (INW), Technical University Graz, began in 1987, initiated by a request from the road maintenance service at the Arlbergpass for an instrument which could verify the results of artificial triggering of avalanches under circumstances where an optical observation was difficult or even impossible, such as darkness, fog or heavy snowfall. The use of microwave radiation will allow reliable observation of avalanches if a frequency is chosen which is strongly reflected by solid or powder snow moving on the ground but is only negligibly attenuated by falling snow or other precipitation, including clouds and fog. Unlike optical observation, such a measurement method is practically unaffected by weather conditions, and it was therefore selected for the task.

The principle of such a radar (which is described in this section) makes it possible to measure the avalanche's velocities, and therefore assists study of the dynamic properties of the observed avalanche, as well as simple detection of natural avalanches and verification of artificially triggered avalanches. This type of avalanche radar is therefore also very interesting for avalanche research (Randeu and others, 1990).

Technically the avalanche radar is a pulsed radar which emits short pulses of microwave energy and analyzes the received echoes reflected from the target objects hit by the antenna beam. In this special case the reflecting object is the ground covered with steady or moving snow.

In addition to simple echo analysis (which is mainly the calculation of the target distance), a pulsed Doppler radar also provides the ability of a continuous-wave (CW) radar - the type of radar so far applied in snow and avalanche warning (Gubler and others, 1986) - to measure velocities by means of the Doppler effect. Due to this physical law, the frequency of the received echo signal is shifted against the transmitted signal, and the frequency change, also called Doppler frequency or shift, is proportional to the reflecting object's velocity relative to the radar antenna.

$$
f_{\text {Doppler }}=\left(2 f_{0} / c\right) v,
$$

where $f_{\text {Doppler }}$ is the Doppler frequency shift, $f_{0}$ is the radar transmission frequency, $v$ is the target radial velocity, and $c$ is the velocity of light.

The combination of these two radar types provides information on how far away the individual echo components originate and how fast the echo-producing targets move. Besides making the detection and verification of avalanches more reliable, this pulsed Doppler radar allows spatiallyresolved dynamic analysis of snow and ice avalanches (e.g. development of speed, front wave speed, acceleration and deceleration characteristics). If a relation between the intensity of the received signal and the volume of the avalanche can be established in future research, this would, together with information about the snow density, allow observation of mass development and energy release and decay.

The range resolution is accomplished by a quasi-simultaneous acquisition of Doppler frequencies from a number of adjacent sections along an avalanche run (see Fig. 1). This process is called range-gating and allows the spatial discrimination of echo intensity, velocity and its spectral distribution in the top-down direction. The length of these range elements is proportional to the duration of the transmitted microwave pulse. The choice of the range-gate length and the limitations on this choice are described in the next section together with other radar parameters.

\section{RADAR SPECIFICATIONS}

A suitable choice of the radar parameters is important for the operation of the radar. The first parameter to mention is the radar microwave frequency $f_{0}$ because it determines, on the one hand, the physical properties like reflectivity and attenuation of the signal and, on the other hand, technical and 


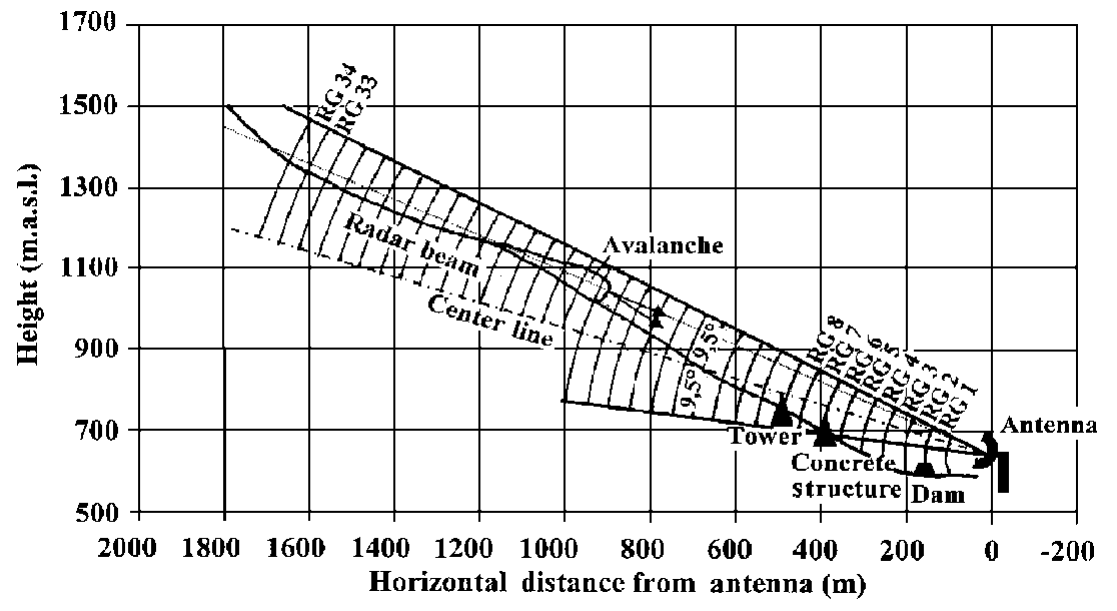

Fig. 1. Vertical profile of the avalanche path in Ryggfonn, together with the radar illumination geometry (RG, range gate).

economical issues. Because of the above-mentioned requirement that the radar be able to operate reliably under bad weather conditions, a frequency in the G-band $(4-8 \mathrm{GHz})$ has been chosen. Due to the comparatively large wavelength $(5.2 \mathrm{~cm}$ at $5.8 \mathrm{GHz}$, which is the frequency used), the signal is hardly attenuated by small particles like raindrops or snowflakes but is reflected sufficiently by the compact snow of avalanches. On the other hand, this allows the detection only of the dense-flow part of avalanches. To detect powder-snow avalanches a higher frequency of about $35-40 \mathrm{GHz}$ would be necessary, and an extension to the existing radar at $35.8 \mathrm{GHz}$ has already been developed.

Another parameter is the so-called pulse repetition frequency $f_{\text {prf }}$ which gives the number of pulses transmitted per second. It determines the maximum measurement range $r_{\max }$ (also called unambiguous range) since the echo reflected by the most distant target must already have been received by the radar before the next pulse can be transmitted.

$$
r_{\max }=\frac{c}{2 f_{\text {prf }}}
$$

where $c$ is the velocity of light.

Since the Doppler signal is sampled with $f_{\text {prf }}$, the maximum Doppler frequency is $f_{\text {prf }} / 2$ according to Shannon's sampling theorem which determines the maximum measurable velocity $v_{\max }$ :

$$
v_{\max }=\frac{c}{2 f_{0}} \frac{f_{\mathrm{prf}}}{2} .
$$

Since sampling with $f_{\text {prf }}$ leads to oversampling (observed avalanche velocities are normally at least eight times lower than $v_{\max }$ ), averaging with its advantages of data and noise reduction is possible, so the maximum measured velocity is reduced by the oversampling factor. The pulse-repetition frequency is adjustable in the range $40-60 \mathrm{kHz}$, so $r_{\max }$ is $3750-2500 \mathrm{~m}$ and $v_{\max }$ (with eight times oversampling) is $65-97 \mathrm{~m} \mathrm{~s}^{-1}$.

The pulse length $\tau$ determines the range resolution of the radar. Although a high resolution with a very short pulse would be preferable, there are limits given by the bandwidth $(\mathrm{B} \sim 1 / \tau)$ and the technical feasibility (need of very fast switches for pulse shaping and fast analogue/digital converters for sampling). Furthermore, shorter pulses transmit less energy and therefore reduce the detection range. The INW avalanche radar has a minimum pulse width of $0.33 \mu \mathrm{s}$, equivalent to a range-gate length of $50 \mathrm{~m}$.
The transmission power of the radar is a trade-off between the detection range and the costs for the amplifier which is the single most expensive part of the radar. A peak power of $5 \mathrm{~W}$ should be sufficient for most cases. When used in 1997 in Ryggfonn, Norway, the radar had an amplifier with only $1 \mathrm{~W}$ output power.

The antenna beamwidth must be chosen in such a way that the whole area of interest is illuminated by the radar.

The number of range gates is not really a parameter of the radar itself but only of the data-acquisition unit. Its choice depends mainly on the local conditions and requirements, and the value is typically $20-40$.

\section{GOMPONENTS OF THE RADAR}

Three avalanche radars have been built so far, and although there are minor differences between these instruments due to different user requirements and various technology updates, their overall structure is the same and has three major components:

antenna: parabolic reflector with a diameter of $0.7-1.8 \mathrm{~m}$ (depending on the purpose of the radar and the required maximum range and illumination width)

$R F$ (radio-frequency) unit: This is a box which is situated directly beneath the antenna to minimize microwave signal attenuation in the cable. It contains the microwave circuits of the transmitter that generates the microwave pulses and the receiver. Also control circuits for the radar and the necessary power-supply units are situated in the RF box.

Data-acquisition and control unit: For the mobile radar used in the Ryggfonn measurement campaign, the components of this unit are also in a transportable box. Its main part is a personal computer which controls all the functions of the radar and also does the necessary digital signal processing. It archives the measured data on a hard disk and displays them on a screen. The maximum distance between this unit and the RF box is about $50 \mathrm{~m}$ (limited by tolerable attenuation due to the cable).

Each component of the transportable radar owned by the Austrian Institute for Avalanche and Torrent Research (AIATR) can be lifted and handled by a single person. To 

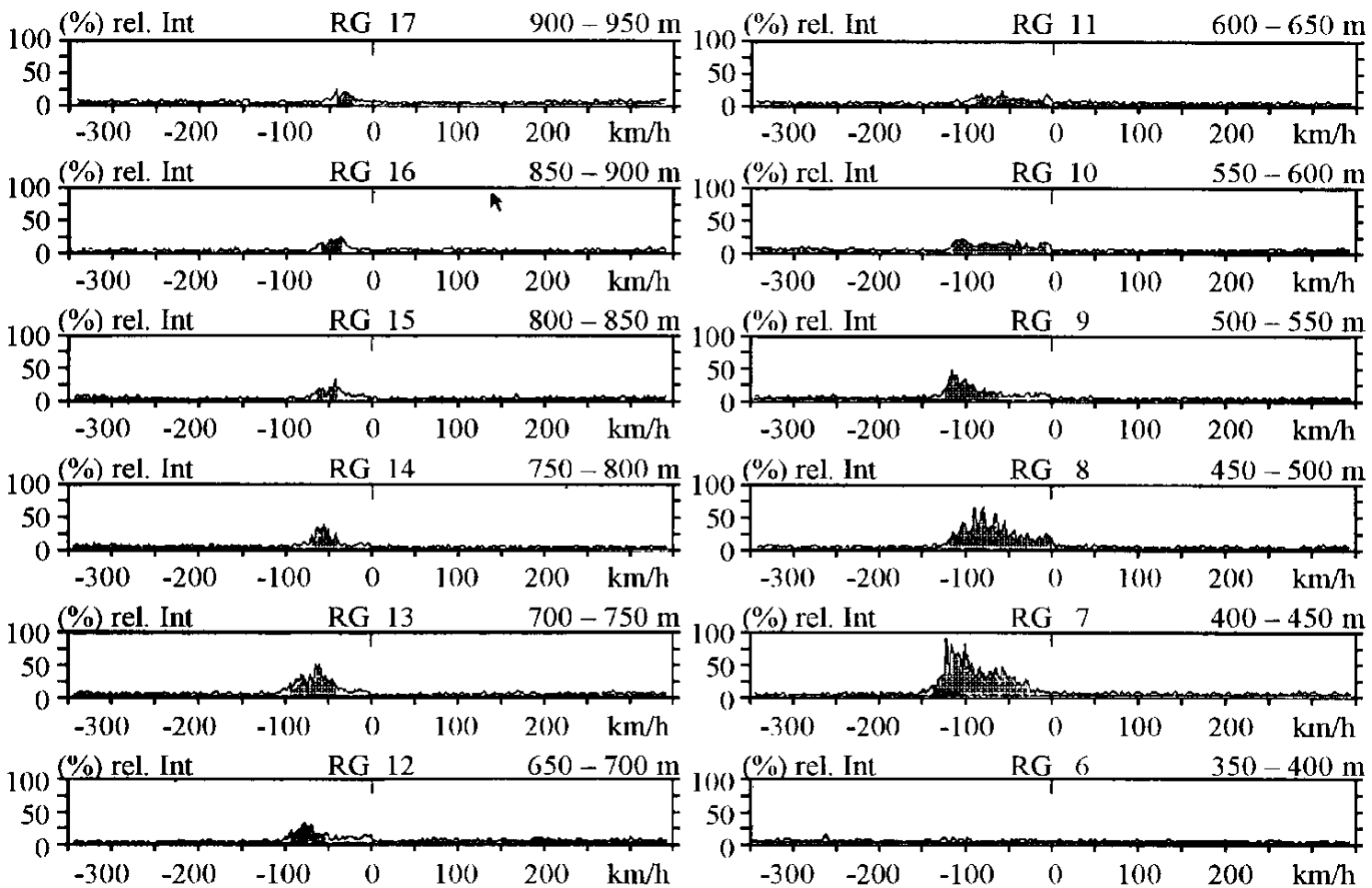

Fig. 2. Screen display of raw velocity spectra; range-gate numbering is bottom-up.

allow safe operation of the electronic devices inside, the box of the RF unit can be heated.

The radar is controlled by software using an easy-tooperate graphical user interface. This allows the display of status information and of the measured data. The radar can be started and stopped by a single mouse click, and the various parameters necessary for the set-up of the radar are adjusted in dialog windows.

\section{MEASURED DATA}

The received echo signal is first converted into a so-called In-phase/Quadrature-phase (= complex) signal and then sampled at instants corresponding to the individual range gates (all range gates are sampled within each pulse cycle). The complex signal allows the calculation of a complex fast Fourier transform (FFT), making it possible to discriminate between positive and negative Doppler frequencies (i.e. velocities). The FFTs, which are performed for all range gates independently, need 2048 samples each (if eight times oversampling is chosen). So a complete calculation of the spectra takes 2048 pulse cycles or $37 \mathrm{~ms}$ (at a pulse-repetition frequency of $55 \mathrm{kHz}$ ). In reality, this value is normally about four times higher due to additional averaging of the spectra. Since this is done for all range gates concurrently, the number of range gates does not affect the measurement time.

The corresponding velocity spectra are displayed on-line by the radar software. Figure 2 shows a typical example of the radar output. The abscissa of the spectra shows the velocity, while the ordinate shows the intensities of the spectral components of the echo signal. As mentioned above, one measurement cycle consists of $n$ simultaneous spectra, where $n$ is the number of configured range gates (abbreviated as RG in the figures). So the radar delivers velocity spectra every 100-200 ms for 20-40 different points spaced equidistantly (50, 100 or $150 \mathrm{~m}$ ) along the avalanche track up to a theoretical maximum range of about $2.5 \mathrm{~km}$. Of course the real measurement distance may be smaller because an amplifier is not powerful enough or because a higher noise floor is produced by clutter (which is not an important issue since the most frequent clutter is produced by stationary or slow-moving objects and can be eliminated easily). The value is also affected by local conditions. Due to the 256 points of the FFT, the distance between the points is about $0.5 \mathrm{~m} \mathrm{~s}^{-1}$ $\left(v_{\max } / 128\right)$. Because of the use of a rectangular time-window (which has a narrow bandwidth but high side lobes) for the FFT with the maximum possible length (all 2048 cycles), the actual velocity resolution is the same as the spacing.

The measured spectra shown in Figure 2 are from the experiment in Ryggfonn. At the moment displayed in the figure, the avalanche front is in RG 7 (400-450 m from the radar antenna) with a peak velocity of about $150 \mathrm{~km} \mathrm{~h}^{-1}$ (in the past the diagrams have been labelled in $\mathrm{km} \mathrm{h}^{-1}$ and not in $\mathrm{m} \mathrm{s}^{-1}$ ). However, there are still fast snow movements at farther distances (RG 8 and higher).

The "roughness" of the spectra is due to the random character of the backscattering and can be smoothed by further averaging.

\section{DATA PROGESSING}

While spectra are calculated and displayed on-line during the measurement, further data processing and evaluation is done offline by additional software tools allow the calculation of the time dependency of the velocity for each range gate and the position and velocity of the avalanche front. These calculations need to have a profile of the avalanche track since a Doppler radar always measures the radial velocity component directed towards the radar antenna, and not the actual velocity along the avalanche track, which is important for dynamic analysis. With knowledge of the profile, the velocity can be corrected to obtain the real value. 


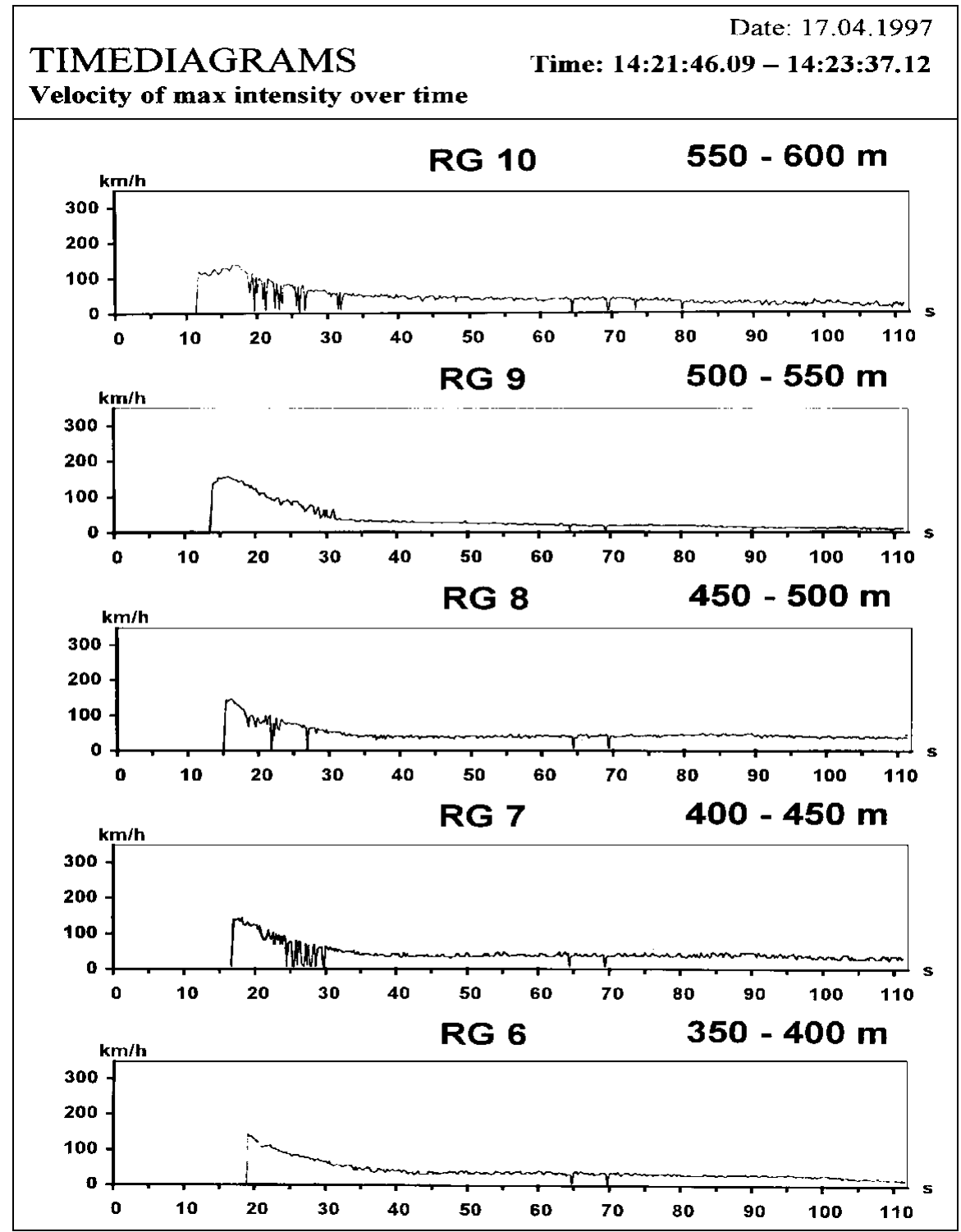

Fig. 3. Time dependency of the avalanche velocity.

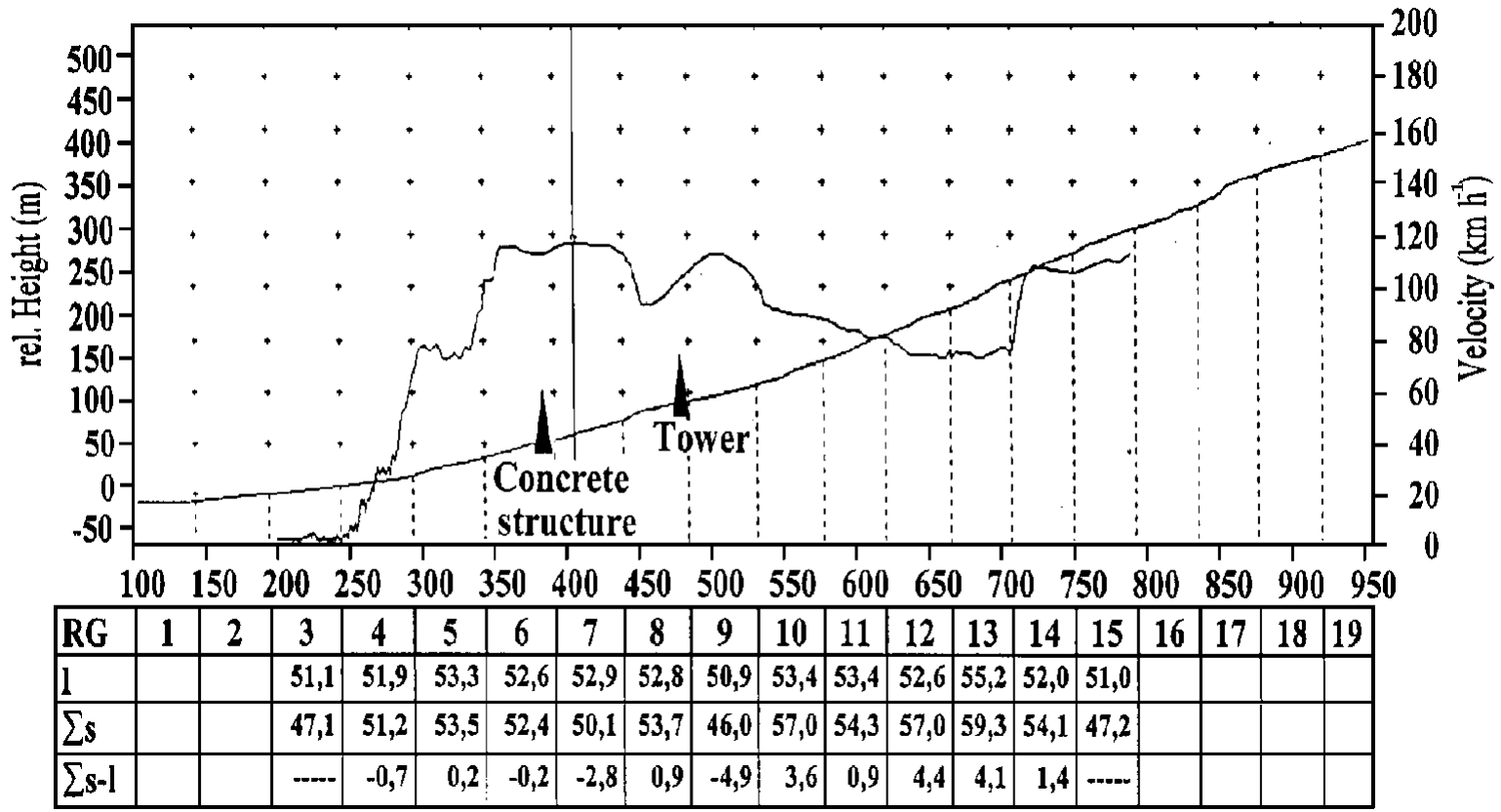

Fig. 4. Front-velocity diagram. 


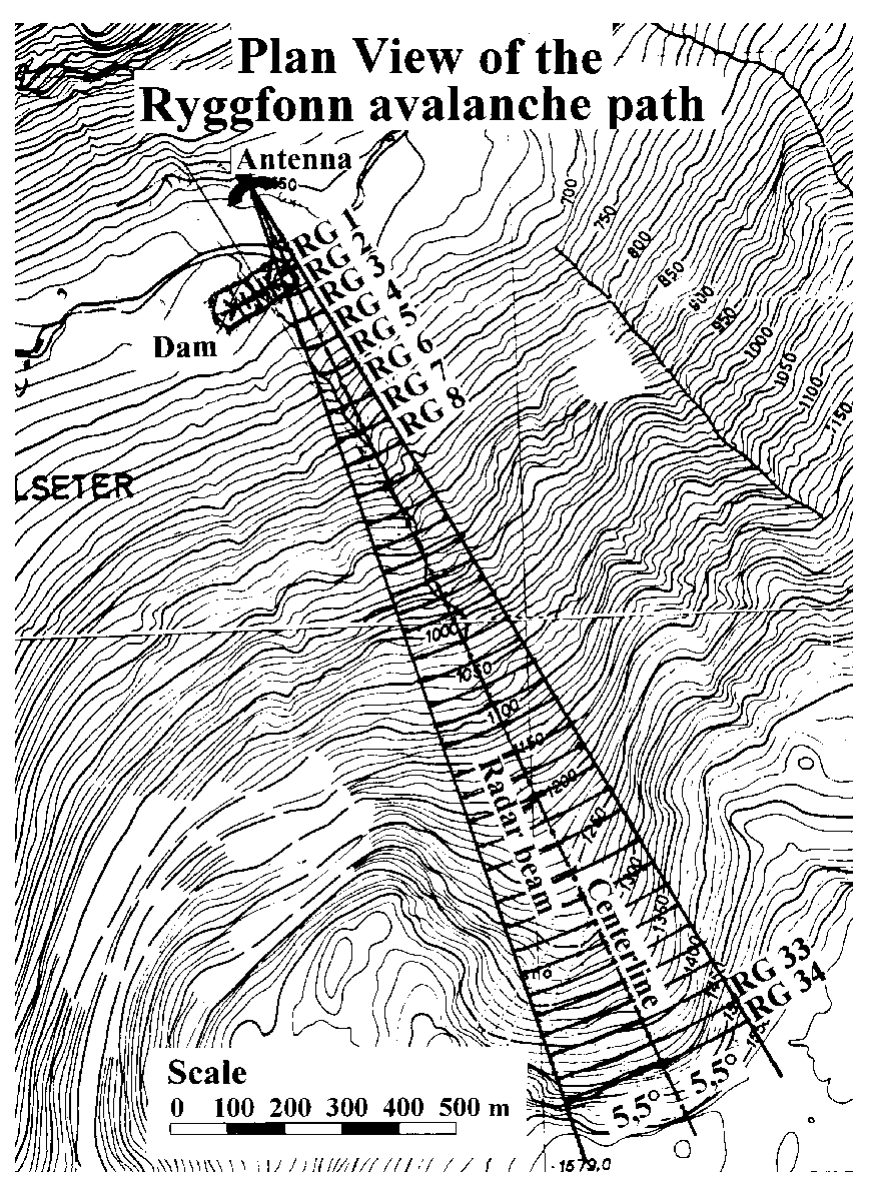

Fig. 5. Plan view of the Ryggfonn avalanche path with radar beam coverage.

There are several possibilities for determining a representative avalanche velocity from the spectra (see Fig. 2), among them:

Velocity of maximum intensity: this is the velocity of the spectrum line with the highest echo intensity.

Maximum velocity: this is the highest velocity occurring in the spectrum for which the echo intensity is above a specified level (normally a multiple of the always present noise level).

Mean velocity: this is an average (weighted by the echo intensity) of the velocity.

These velocities must be determined separately for each range gate and each time-frame, and the results are twodimensional diagrams (see Fig. 3). The abscissa in these diagrams shows the elapsed time since the beginning of the measurement, while the ordinate displays the corresponding velocity. The time diagrams show the development of the selected representative velocity in each range gate, while the spectral distribution is neglected. This data reduction (compared to the spectra) makes it easier to determine some interesting parameters like

the instant the avalanche enters a range gate

the run time needed by the avalanche to cover a range gate

the maximum value of the velocity in each range gate

the overall maximum velocity of all range gates.

Another interesting result which can be derived from the measured data is the so-called front-velocity diagram. It shows the velocity of the avalanche front in dependence on its position along the track (see Fig. 4). In this diagram, the abscissa shows the horizontal distance from the antenna, and the ordinate the profile (relative height above the antenna) of the track and the velocity. The front velocity is calculated from the time diagrams in the following way: Looking at Figure 3, the moment when the avalanche enters a range gate can be easily determined, and the position at this time obtained from the profile. Since the velocity and the next time-frame are known, the distance covered by the avalanche front during this short interval (normally 100-200 ms) can be calculated by the following formula:

$$
d_{i, i+1}=\left(t_{i+1}-t_{i}\right) \frac{v_{i+1}+v_{i}}{2}
$$

This is an iterative process starting at the instant the avalanche enters the range gate (time $t_{0}$, position $P_{0}$ ). With knowledge of the avalanche track profile, the next front position $P_{1}$ is determined by moving the above calculated distance $d_{0,1}$ along the assumed path. From $P_{1}$ we obtain $P_{2}$ by calculating $d_{1,2}$ and so on, up to the time-frame at which the avalanche enters the adjacent lower range gate. To obtain the whole front-velocity diagram, this calculation must be repeated for every range gate. The quality of these calculations can be determined by comparing the sum of the distances calculated in the way described with the actual length of the avalanche path in the given range gate (which is derived from the topographical data). Since these values are most likely not equal for the first calculations it will be necessary to repeat the whole process with an optimized choice of velocities. These distances and their difference are shown in the table below the diagram (see Fig. 4).

By this method the front position can be determined with a precision depending on the time interval between the measurement cycles and on the quality of the profile data. This is normally much better than the spatial resolution of the radar itself, which is the range-gate length (minimum $50 \mathrm{~m}$ ).

\section{MEASUREMENT AT RYGGFONN}

A plan view of the experimental site of the Norwegian Geotechnical Institute (NGI) at Ryggfonn is shown in Figure 5. The corresponding profile of the avalanche path can be seen in Figure 1. Permanently installed measurement instruments (e.g. pressure sensors) are located on an instrument tower (situated in RG 8) and on a concrete structure (RG 6). There is also a dam at the end of the avalanche path.

The radar set-up for the measurement consisted of 34 range gates with a length of $50 \mathrm{~m}$ each, starting at $100 \mathrm{~m}$ from the antenna. This would give a theoretical maximum measurement range of $1800 \mathrm{~m}$. Unfortunately this value has been cut to an actual distance of $900 \mathrm{~m}$, from which good results could be obtained because the upper part of the track beginning at a distance of about $1200 \mathrm{~m}$ was not illuminated by the antenna beam due to the profile's curvature. Additionally, the microwave power of $1 \mathrm{~W}$ was not sufficient for this distance - it has been upgraded to $5 \mathrm{~W}$ in the meantimeand the antenna was probably not optimally aligned.

The experiment took place on 17 April 1997 in the early afternoon. The avalanche was released by explosives. The amount of new-snow accumulation during the week before release was $15 \mathrm{~cm}$. On 16 April, the day before the experi- 
ment, the air temperature rose to above freezing below $1200 \mathrm{~m}$ a.s.l. At the time of the avalanche the $0^{\circ} \mathrm{C}$ isotherm was about $1000 \mathrm{~m}$ a.s.l. Initially a dry slab was released by the explosion which entrained gradually moister surface snow on its way down the track. The deposit of the avalanche consisted of partly wet snow blocks with an average density of $540 \mathrm{~kg} \mathrm{~m}^{-3}$ measured from the uppermost $150 \mathrm{~cm}$ in a sample pit. The estimated volume was approximately $40000 \mathrm{~m}^{3}$.

Because of the above-mentioned problems the radar measurement and data recording were started only when the avalanche entered RG 15 (800-850 m).

Figure 2 shows a typical diagram of the raw velocity spectra (about $18 \mathrm{~s}$ after the beginning of the measurement). The time diagrams derived from these data are shown in Figure 3. Due to the wet snow of the avalanche, a very long after-running at low velocities, after the passing of the avalanche front, occurred as seen in these diagrams.

The last step of the data analysis was the determination of the corrected front velocity. The results of these calculations are shown in Figure 4. The table at the bottom shows that the discrepancy between the real and the calculated run length is $4.9 \mathrm{~m}$ in the worst case, which is $<10 \%$ of the range-gate length. The velocity plot starts in RG 15 with a velocity of about $110 \mathrm{~km} \mathrm{~h}^{-1}$. After a decrease to $75 \mathrm{~km} \mathrm{~h}^{-1}$ in RG 13 the speed increases again and reaches about $112 \mathrm{~km} \mathrm{~h}^{-1}$ in RG 9. A short slowdown in RG 8 because of a smaller slope angle is followed by a velocity maximum of $117 \mathrm{~km} \mathrm{~h}^{-1}$ (or $32.5 \mathrm{~m} \mathrm{~s}^{-1}$ ) in RGs 7 and 6 where the avalanche track is more channelled. After that, the avalanche comes to a halt very quickly before the end of RG 3. This occurs close to the above-mentioned dam.

The shear and moment strain measurements in the steel tower situated in RG 8, and the pressure measurements by load cells located on the structure in RG 6, allowed verification of the velocity measurements by the radar. A time difference of $2.9 \mathrm{~s}$ between the first measured impacts on the tower and the concrete structure and a distance of $103 \mathrm{~m}$ gives an average velocity of $35.5 \mathrm{~m} \mathrm{~s}^{-1}$. The average front velocity between these two obstacles calculated from the diagram in Figure 4 is $108 \mathrm{~km} \mathrm{~h}^{-1}$, or $30 \mathrm{~m} \mathrm{~s}^{-1}$. The discrepancy between these two values can be accounted for by a closer examination of the impact pressure at the load cells: $0.57 \mathrm{~s}$ after the first impact with a peak of $52 \mathrm{kPa}$, a second, higher peak occurs. Photos of the avalanche front show a cloud of powder and flying snowballs with a low density before the dense flow part. While the cloud would be responsible for the first pressure peak, the dense part has produced the second peak. As described earlier, the radar can detect only the later part of the avalanche. If we take the time difference of $3.47 \mathrm{~s}$ between the impacts into the tower and the concrete structure we obtain an average velocity of $29.7 \mathrm{~m} \mathrm{~s}^{-1}$. These measurements seem to verify the calculated front velocity, and although some circumstances, as mentioned above, were not optimal, the amount of data acquired by this experiment and the results of the analysis were satisfactory.

\section{SIGNALLING AND WARNING}

Besides its scientific task of measuring the dynamic properties of avalanches, the radar is also suited for reliably detecting avalanches which endanger traffic corridors. It can be used for signalling purposes and for closing roads (or railways) if the avalanche run length, in relation to the length of the traffic corridor affected, provides sufficient signalling lead time, i.e. the time-span between unambiguous, reliable detection of an emerging dangerous event and its arrival at the passenger zone.

The main advantage of the pulsed Doppler radar is the data processing, making it possible to discriminate between avalanches and other events (e.g. skiers, snowdrift by wind) which may erroneously trigger other types of detectors. Another advantage is that the instrument need not be situated in the avalanche track but may be safely placed close to the end of the run-out zone, so access for maintenance in winter conditions is no problem.

\section{CONGLUSIONS}

Pulsed Doppler avalanche radar provides information about the avalanche velocity and its spatial development and distribution - although there has not been much research into the interpretation of the velocity spectra till now - along the track. It thus differs from most other instruments, which provide information about the parameters of interest (e.g. velocity, pressure) of an avalanche either only at a single selected location in the path, or else from the whole path but without the possibility of spatial discrimination.

Data from a pulsed Doppler radar can be used to verify and improve avalanche models. This will make it attractive since many of the newer models outputs are also velocity fields $v(x, t)$, so a comparison between the measurements and the results of the models should be straightforward, although the computer simulations will have a better range resolution and may be delivering two- or three-dimensional data which the radar cannot provide.

What can be done on snow avalanches can also be done on slush or debris flow with this radar, and even the measurement of water-speed profiles in rivers would be an easy task. Finally, the sophisticated, and to some degree redundant, data interpretation makes such radar a highly reliable tool for pure detection and signalling, clearly discriminating an avalanche from other moving objects like skiers, cable cars and trees in the wind.

\section{ACKNOWLEDGEMENTS}

Thanks are expressed to K. Kristensen and K. Lied from the NGI for their contributions to carrying out this experiment.

\section{REFERENGES}

Gubler, H., M. Hiller, G. Klausegger and U. Suter. 1986. Messungen an Fliesslawinen. Zwischenbericht 1986. Eidg. Inst. Schnee- und Lawinenforsch. Mitt. 41.

Randeu, W. L., R. Okorn and W. Riedler. 1990. A pulsed Doppler radar for the acquisition of avalanche dynamics. In CIV'90 Conference on Avalanches and Planning of Mountain Territory, October 1990, Arabba, Italy. Proceedings. Arabba, Dipartimento Foreste. Centro Sperimentale Valanghe e Difesa Idrogeologica, 118-126. 